\title{
Natural chelators for prevention of diseases
}

Syed Anzar Ahmad Rizvi ${ }^{1}$, Alvina Tariq ${ }^{2}$, Amna Anzar ${ }^{3}$ and Kisa Fatima Altaf 4* $^{*}$

1. Dow University of Health Sciences, Department of Community Medicine, Karachi-Pakistan

2. Dow University of Health Sciences, Department of Undergraduate Skills Lab, Karachi-Pakistan

3. Jinnah Sindh Medical University, Department of Pharmacology, Karachi-Pakistan

4. Dow University of Health Sciences, Department of Professional Development Centre, Karachi-Pakistan

*Corresponding author's email: kisafatima3@gmail.com

Citation

Syed Anzar Ahmad Rizvi, Alvina Tariq, Amna Anzar, Kisa Fatima Altaf. Natural chelators for prevention of diseases. Pure and Applied Biology. Vol. 7, Issue 2, pp575-578. http://dx.doi.org/10.19045/bspab.2018.70071

\begin{tabular}{llll}
\hline Received: 27/02/2018 & Revised: 18/04/2018 & Accepted: 28/04/2018 & Online First: 03/05/2018 \\
\hline
\end{tabular}

\section{Abstract}

Public health surveys in recent years have demonstrated a burden of diseases caused due to oxidative stress and inflammation. Disease caused by oxidative stress has been observed to be cured by using natural chelators. This paper describes various chelators and their properties in treating multiple diseases. Literature for this review article was collected from different sources, to capture as many relevant references as could reasonably be expected, an extensive variety of medical, Ecological, environment and scientific databases were looked to distinguish essential Investigations. Moreover, different web engines were hunt down site pages that may give references. The aim of this review article is to evaluate and highlight the importance of natural chelators identified and their importance in prevention of diseases as it is one of the most neglected approach in medical practice. Relevant research article of the last fifteen years were Reviewed especially from PubMed but some article up to twenty years were also included for Having interesting content. A higher therapeutic index of natural chelators has been accounted with treating different neurodegenerative diseases, skin diseases and multiple stages of cancers. Additional clinical research is needed to be done to make results more clear, physiological and molecular alterations of diseases are needed to be evaluated in order to select an appropriate chelator and extraction from various fruits and vegetables are needed to be done to get an expertise on these diseases.

Keywords: Chelators; Oxidative Stress; Neurodegenerative disease

Iron chelation in Neurodegenerative diseases

Naturally occurring chelators are being observed to be therapeutically helpful in protection against the diseases caused due to oxidative stress. Many of the research studies are already being done to evaluate the therapeutic index of natural chelators against skin diseases, neurodegenerative disease and cancerous cells [1]. Iron chelation therapies are being induced to prevent against the diseases caused due to iron overload and different types of cancers [1]. They are safe, non-invasive, affordable and available without prescriptions as they are considered food supplements.

The neurodegeneration in much of the neurodegenerative diseases like Parkinson's 
and Alzheimer's are multifactorial, which depends upon the complexity of molecular toxic reactions, inflammation, oxidative stress and generation of protein aggregates in body which may lead to termination of neuronal activities. Whereas, accumulation of iron in glial cells further worsens the condition [1]. Iron chelation and antioxidant therapies are observed to be effective against oxidative stress pathogenesis in neurodegenerative diseases. Deregulation of brain's iron metabolism can lead to generate early pathological events for Alzheimer's disease [2]. These may include inflammation, oxidative stress and failure in neuronal cell cycle regulation; this might ultimately lead to cellular apoptosis. Researchers have evaluated a direct link between Alzheimer's disease neurodegeneration and iron metabolism; these results have led to the production of iron chelating neuroprotective drugs affecting different targets in brain [3]. Catechins (tea flavonoids) are being demonstrated in protection against neurodegeneration by promoting metal chelating, antioxidant and anti-inflammatory properties [4]. Much of the research is being done in animal studies to evaluate the neurodegeneration, these compounds are observed to cross the blood brain barrier in order to prevent or reduce neuronal cell death [3].

\section{Prevention of skin damage by natural phenols}

Human skin is continuously exposed to ultraviolet radiations; this causes a wide number of pathological changes in skin texture, ultimately leads to edema or tumor cell formation. Skin damage is commonly observed due to the exposure of ultraviolet radiations, a long term exposure to UV radiations may cause a variety of biological changes which include DNA damage, erythema, edema, hyperplasia, photoaging and melanogenesis. This alteration ultimately turns to skin cancer $[5,6]$. UV radiations are considered a potent modulator of molecular phytochemical reactions; it causes excitation of electrons, which then causes chemical medications in reactive molecules. When a molecule absorbs UV radiation, it destroys itself and other neighboring molecules by producing Reactive oxygen species (ROS). The absorbed UV radiations react intracellularly with photosensitive molecules, these reactions then produce cytotoxic and genotoxic effects [7]. ROS are developed in skin when exposed to ultraviolet radiations; these ROS are believed to alter the body metabolism by reacting with DNA, proteins and fats, which causes oxidative damage to the body. The oxidative damage to body may cause certain harmful events like altered cellular metabolism, altered regulation pathways, morphological changes, cell differentiation, proliferation and apoptosis in texture of skin. These disturbances may cause aging and tumor cell formation on skin. The harmful ultraviolet effects on skin can be prevented by using antioxidants. Currently skin care specialist declared that natural occurring herbal compounds like phenolic acids, flavonoids and phenols with higher molecular weight can be used to prevent skin from getting damaged [8].

Around 8000 naturally occurring phenolic compounds are being found, all of them contains a phenol $[10,11]$. The naturally occurring phenolic compounds can be found in herbs, tea and coffee beans. Phenolic compounds can also be found in some fruits like apple, peaches, apricots, citrus fruits, grapes, cherries, berries and plum, some vegetables are also discovered with the presence of phenolic compounds like lettuce, broccoli, tomatoes, onion, olives, spinach, cabbage and plants [9-12]. All the naturally occurring phenolics contain two consecutive carbon frameworks the hydroxycinnamic and hydroxybenzoic acids [13]. A variety of beneficial biological activities are being 
observed in mammals by using different phenolic compounds, these mixes can supportive in keeping a few diseases as they fill in as antiviral, antibacterial, antithrombotic, mitigating and anticarcinogenics $[9,10]$.

\section{Beneficial properties of different phenolic} Phenolic are believed to prevent DNA damage by acting in redox sensitive cascades [14]. Flavonoids and phenolic acids are being provided with antioxidant herbal properties; therefore, it is being observed that phenolics are helpful in preventing UV induced production of oxygen free radicals in skin. Caffeic acid and ferulic acid are mostly present in grains, fruits and vegetables, these are observed in preventing phospholipids membranes from UV radiation, commonly observed to prevent against UV induced erythema [15]. Cosmetologists normally induce ferulic acids in sunscreens and skin lotions, as they are observed being best absorber of UV radiation on skin [20]. Quercetin is another form naturally occurring flavonoids, commonly found in fruits, vegetables, olive oils and bee hives, these are found be strong metal chelators with antioxidant properties. Quercetins are found to be effective against UV radiation by reducing its antioxidant properties [16].

Apigenin, a plant flavonoid found in herbs are observed to serve as anticarcinogens, these are beneficial in preventing tumor formation [17]. Genistein are most commonly found in soy beans, these are being observed in altering the cell cycle and preventive against many types of cancers. Genistein are believed to be preventive against contact hypersensitivity and inflammatory edema formation in skin [18]. Resveratrol are most commonly found in grape seeds, peanuts and some berries, it prevents proliferation by demonstrating its antioxidant and antiinflammatory properties [3]. Resveratrol are also demonstrated with its anti-tumor and anti-cancerous

properties

[17].
Nordihydroguaiaretic acid was tested as a chemo protective drug for animal studies. Carnosic acids are found in rosemary and sage observed to possess their antioxidant properties, its chemoprotective effects are tested in animal studies. Tannins are found in different plants; they serve as a strong protector against antioxidants and prevent free radical damage against the exposure of UV radiations. Tannins are demonstrated to be protective against premature skin aging and formation of cancerous cells on skin [19]. Most of the natural chelators occurring in different fruits and vegetables and herbs are already being encountered in skin lotions and sunscreens; however most of these compounds are tested in animal studies and are still awaited to be evaluated and tested on human skin.

\section{Conclusion}

Natural chelators are now being evaluated with their therapeutic values against many diseases. In recent years most of these are observed in treating neurodegenerative diseases, skin diseases and in many types of cancers as a chemoprotective agent. It is important to recognize the molecular alterations occurring in different diseases in order to select a precise chelator. A wide variety of phenolics are being identified with their therapeutic effects against skin diseases.

\section{Authors' contributions}

Conceived the idea: SAA Rizvi, Corrections: SAA Rizvi, Proof Reading: A Anzar \& A Tariq, Correspondence: KF Altaf, Wrote the paper: KF Altaf

\section{References}

1. Weinreb O, Mandel, S, Youdim, MB \& Amit $\mathrm{T}$ (2013). Targeting dysregulation of brain iron homeostasis in Parkinson's disease by iron chelators. Free Radical Biology and Medicine 62: 52-64.

2. Butterfield, DA, Di Domenico F \& Barone E (2014). Elevated risk of type 2 diabetes for development of Alzheimer disease: a key role for oxidative stress in brain. Biochimica et 
Biophysica Acta (BBA)-Molecular Basis of Disease 1842(9): 1693-1706.

3. Mandel S, Amit T, Reznichenko L, Weinreb O \& Youdim MB (2006). Green tea catechins as brain-permeable, natural iron chelatorsantioxidants for the treatment of neurodegenerative disorders. Molecular Nutrition \& Food Research, 50(2): 229-234.

4. Anand, P, Kunnumakara AB, Sundaram C, Harikumar KB, Tharakan ST, Lai OS \& Aggarwal BB (2008). Cancer is a preventable disease that requires major lifestyle changes. Pharmaceutical Res 25(9): 2097-2116.

5. Tebbe B (2001). Relevance of oral supplementation with antioxidants for prevention and treatment of skin disorders. Skin Pharmacology and Physiology 14(5): 296-302.

6. Afaq F \& Mukhtar H (2001). Effects of solar radiation on cutaneous detoxification pathways. Journal of Photochemistry and Photobiology B: Biology 63(1-3): 61-69.

7. De Gruijl FR (2002). Photocarcinogenesis: UVA vs. UVB radiation. Skin Pharmacology and Physiology 15(5): 316-320.

8. Svobodová A, Psotová J \& Walterová D (2003). Natural phenolics in the prevention of UV-induced skin damage. A review. Biomed Pap Med Fac Univ Palacky Olomouc Czech Repub 147(2): 137-145.

9. Ross, JA \& Kasum CM (2002). Dietary flavonoids: bioavailability, metabolic effects, and safety. Ann Rev of Nutrition 22(1): 19-34.

10. Kris-Etherton PM, Hecker KD, Bonanome A, Coval SM, Binkoski AE, Hilpert KF \& Etherton TD (2002). Bioactive compounds in foods: their role in the prevention of cardiovascular disease and cancer. The American J of Medicine 113(9): 71-88.

11.Rechner AR, Spencer JP, Kuhnle G, Hahn U \& Rice-Evans CA (2001). Novel biomarkers of the metabolism of caffeic acid derivatives in vivo. Free Radical Biology and Medicine 30(11): 1213-1222.

12. Birt DF, Hendrich S \& Wang W (2001). Dietary agents in cancer prevention: flavonoids and isoflavonoids. Pharmacology \& Therapeutics 90(2-3): 157-177.

13. Robbins RJ (2003). Phenolic acids in foods: an overview of analytical methodology. $J$ of Agricultural and Food Chemistry 51(10): 2866-2887.

14. Rice-Evans C, Spencer JP, Schroeter H \& Rechner AR (2000). Bioavailability of flavonoids and potential bioactive forms in vivo. Drug Metabolism and Drug Interactions 17(1-4): 291-310.

15. Saija A, Tomaino A, Trombetta D, De Pasquale A, Uccella N, Barbuzzi $\mathrm{T} \&$ Bonina $F$ (2000). In vitro and in vivo evaluation of caffeic and ferulic acids as topical photoprotective agents. Inter $J$ of Pharmaceutics 199(1): 39-47.

16. Erden Inal M, Kahraman A \& Köken $T$ (2001). Beneficial effects of quercetin on oxidative stress induced by ultraviolet A. Clinical and Experimental Dermatology 26(6): 536-539.

17. Afaq, F., \& Mukhtar, H. (2002). Photochemoprevention by botanical antioxidants. Skin Pharmacology and Physiology 15(5): 297-306.

18. Salvi M, Brunati AM, Clari G \& Toninello A (2002). Interaction of genistein with the mitochondrial electron transport chain results in opening of the membrane transition pore. Biochimica et Biophysica Acta (BBA)-Bioenergetics 1556(2-3): 187196.

19. Gali-Muhtasib HU, Yamout, SZ \& Sidani, MM (2000). Tannins protect against skin tumor promotion induced by ultraviolet-B radiation in hairless mice. Nutrition and Cancer 37(1): 73-77.

20. Saija A, Tomaino A, Cascio RL, Trombetta D, Proteggente A, De Pasquale A \& Bonina F (1999). Ferulic and caffeic acids as potential protective agents against photooxidative skin damage. $J$ of the Sci of Food and Agri 79(3): 476-480. 